\title{
CALCULS POUR L'OUVERTURE DE LA BOUCHE DE LA TERRE: ÉTUDE DU TEMPS, GÉOMANCIE ET ART DIVINATOIRE AU LADAKH
}

\author{
Pascale Dollfus
}

\begin{abstract}
À Sonam Gompo
Que poussent des plants turquoise aux fruits d'or! Que poussent des épis doubles aux fruits doubles!

Des épis copieux et sucrés!

Que deux en donnent mille!

Qu'un grain en donne cent!

Par le Lama et par les Trois Joyaux,

Qu'une grande fête soit donnée pour le fils, et un beau mariage offert à la fille!

Ô Bouche de la Terre, ouvre-toi!

Ô Bouche de la Terre, ouvre-toi!
\end{abstract}

Et que, couchés, ils couvrent le champ d'un bout à l'autre

Et qu'ils remplissent un grenier de cent et un grenier de mille khal! ${ }^{1}$

À l'annonce du printemps, le rituel du premier labour fête par ces prières le réveil de la terre endormie. ${ }^{2}$ Autrefois, il revenait au roi du Ladakh, maître de la terre et garant de l'ordre à travers le royaume, de tracer le premier sillon dans un champ proche du palais. Par ce geste, il levait l'interdit qui pesait sur le travail de la terre pendant le long et rigoureux hiver, et inaugurait la saison agricole. Cette époque est aujourd'hui révolue, mais le rituel royal du premier labour perdure ${ }^{3}$ et, comme hier, c'est à un astrologue de l'ancienne capitale d'en choisir le jour et l'heure, les protagonistes - hommes et animaux, l'endroit précis du champ où le soc ouvrira la terre et la direction dans laquelle sera

\footnotetext{
${ }^{1}$ Unité de volume, environ $13.5 \mathrm{~kg}$.

${ }^{2}$ Pour une description du rituel du premier labour au Ladakh central, voir Dollfus (1990, 1996); au Zanskar voisin, voir Riaboff (2000).

${ }^{3}$ Région de langue et de culture tibétaine située aux confins occidentaux du Tibet, le Ladakh fut un royaume indépendant jusqu'à sa conquête au milieu du XIX ${ }^{\mathrm{e}}$ siècle par les troupes de Gulab Singh, raja dogra du Jammu. Intégré à l'Union indienne en 1947, il couvre aujourd'hui deux districts de l'état indien du Jammu-et-Cachemire et comptait quelque 200,000 habitants, outre d'importantes garnisons militaires, au recensement de 2001.
} 
tracée la raie. Toutes les circonstances favorables doivent être réunies, car du bon déroulement de l'ouverture de 'la Bouche de la Terre' (sa kha) dépendent les futures récoltes et l'avenir du pays.

Si l'on exclut l'établissement d'horoscopes, peu a été écrit à ce jour sur l'astrologie telle qu'elle est pratiquée au quotidien dans le monde tibétain. Je me propose d'en donner ici un exemple concret en suivant un astrologue ladakhi dans ses calculs, ses hésitations, et ses choix. 'Astrologue en lignée' (brgyud pa'i dbon po), réputé autant pour la justesse de ses prédictions que pour son étonnante capacité à boire, Sonam Gompo a acquis auprès de son père la maîtrise des méthodes de comptes et de calculs (rtsis), un savoir traditionnellement réservé aux hommes. En ce jour de mars 1997, il dispose pour l'aider dans sa tâche d'un recueil de textes astrologiques connu sous le nom de Dartsi ('bras rtsis, 'Calcul des conséquences'), ${ }^{4}$ d'almanachs et d'aide-mémoire.

Au Ladakh, les astrologues ou 'onpo' (dbon po $)^{5}$ établissent le thème astral des nouveau-nés, vérifient le profil astrologique des futurs époux et étudient la compatibilité de leurs horoscopes. Ils déterminent également la meilleure manière de procéder lors d'un décès, fixent les dates favorables pour entreprendre les travaux agricoles ou tout autre événement important (voyage, inauguration de maison, mariage, etc.), décident des rituels à réaliser pour contrer les mauvaises influences et, souvent, se chargent de les accomplir. Ils sont ainsi les spécialistes du rituel des 'Quatre Cents' (brgya bzhi), exorcisme très répandu caractérisé par la fabrication de quatre centaines d'éléments (effigies, lampes à beurre, etc.), comme du rituel de clôture de 'la Porte de la Terre et de la Porte du Ciel' (sa sgo gnam sgo), exécuté pour barrer le passage aux démons qui se lèvent du sol ou descendent des régions célestes.

Laïcs ou membres du clergé bouddhiste, les astrologues officient à la demande de donateurs privés, s'installant alors plutôt dans la pièce du foyer que dans l'oratoire familial, ou pour la communauté dans son ensemble, comme ici pour l'ouverture de 'la Bouche de la terre'.

\footnotetext{
${ }^{4}$ Son titre complet est 'Bras rtsis gsar phreng kun dga'i snying nor, rendu en anglais par 'Results of the conjugation of planets and stars'. Dans cet article, nous renvoyons à l'édition de Thubstan Shanfan publiée à Leh (Ladakh) en 1985.

${ }^{5}$ En tibétain, le terme $d b o n$ po désigne un tantriste (syn. sngags pa).
} 


\section{Calendrier et Décompte du Temps}

Référence des astrologues du Ladakh, l'astrologie tibétaine emprunte à l'astrologie chinoise, désignée par l'expression 'calculs noirs' (nag rtsis), et à l'astrologie indienne, connue sous le nom de 'calculs blancs' (dkar rtsis). ${ }^{6}$ C'est non seulement un art divinatoire, mais aussi une étude du temps, de la chronologie et du calendrier. ${ }^{7}$ Le calendrier tibétain est fondé sur un cycle de douze ans désignés chacun par un signe animal, lo rtags (Souris, Bœuf, Tigre, Lièvre, Dragon, Serpent, Cheval, Mouton, Singe, Oiseau, Chien et Porc) couplé avec l'un des cinq éléments cosmologiques chinois, 'byung lnga (Bois, Feu, Terre, Fer et Eau). Chaque année est composée de douze mois lunaires synodiques $(z l a),{ }^{8}$ numérotés selon leur rang et subdivisés en deux quinzaines 'montante' ( yar ngo) et 'descendante' (mar ngo), comprenant chacune quinze jours lunaires (tshes zhag). Pour faire correspondre le mois lunaire et la suite des jours, certains jours sont supprimés (chad), d'autres répétés (lag), et un mois intercalaire (zla bshol) est ajouté tous les trente-deux mois et demi. ${ }^{9}$

Les semaines comptent sept jours nommés d'après les planètes $\left(g z a^{\prime}\right)$ qui les gouvernent: Lundi est le Jour de la Lune ( $\left.g z a^{\prime} z l a b a\right)$; Mardi, le Jour de Mars ( $g z a^{\prime}$ mig dmar); Mercredi, le Jour de Mercure ( $g z a^{\prime}$ ' lhag $p a$ ); Jeudi, le Jour de Jupiter ( $g z a$ ’ phur bu); Vendredi, le Jour de Vénus ( $g z a^{\prime}$ pa sang); Samedi, le Jour de Saturne ( $g z a^{\prime}$ spen $p a$ ); Dimanche, le Jour du Soleil ( $g z a^{\prime}$ nyi $\left.m a\right)$. Chaque jour est divisé en douze doubles heures, dus tshod bcu gnyis, désignées par l'animal du cycle duodénaire à laquelle cette période du jour est associée. Entre 17 et 19 heures (heure solaire), le coucher du soleil ou 'soleil à l'ouest' (nyi nub), est ainsi connu comme la double-heure de l'Oiseau.

Au Ladakh, ce calendrier luni-solaire est calculé chaque année par Thupstan Shanfan (Thub bstan gzhan phan), un astrologue originaire du Bas-Ladakh tenant boutique à Leh, lequel le publie sous la forme d'un almanach, accompagné de différentes indications d'ordre astronomique, météorologique et pratique. Cette 'chronique de l'année', lo

${ }^{6}$ Ces dénominations renvoient aux noms tibétains donnés respectivement à la Chine et à l'Inde: rgya nag, 'Étendue noire' et rgya dkar, 'Étendue blanche'.

7 Sur l'astrologie tibétaine, les principaux composants du calendrier et de la chronologie, je renvoie au livre de Cornu (1999) et à l'article de Berzin (1997).

${ }^{8}$ De fait, les mots 'mois' et 'lune' se confondent.

${ }^{9}$ Une année solaire compte environ 365.25/366 jours tandis que 'l'année lunaire' lui correspondant se compose de douze ou treize lunaisons, soit 336 ou 384 jours. Sur le calendrier luni-solaire tibétain, voir Lafitte (1985). 
tho ${ }^{10}$ ne commence pas au début de l'année tibétaine officielle, ${ }^{11}$ mais le premier jour du troisième mois tibétain (bod zla), ou mongol (hor zla), vers la mi-avril, à partir de l'entrée du soleil dans le signe du Bélier.

\section{La Chronique de L’année, Lo Tho}

L'almanach publié par Thupstan Shanfan ne paie pas de mine. Il se présente sous la forme d'un livret oblong (dim. $24 \mathrm{~cm} \times 8,5 \mathrm{~cm}$ ) comprenant une centaine de feuillets imprimés en noir et blanc sur du mauvais papier et agrafés ensemble sur leur largeur.

La première partie fournit un mémento chronologique (bstan rtsis) des événements significatifs qui ont eu lieu depuis l'apparition du Bouddha Shakyamuni en Inde, ${ }^{12}$ des prévisions générales concernant la météorologie et les récoltes, illustrées par le dessin d'un bouvier (glang $r d z i$ ); des conseils à suivre en fonction du sexe et de l'année de naissance de

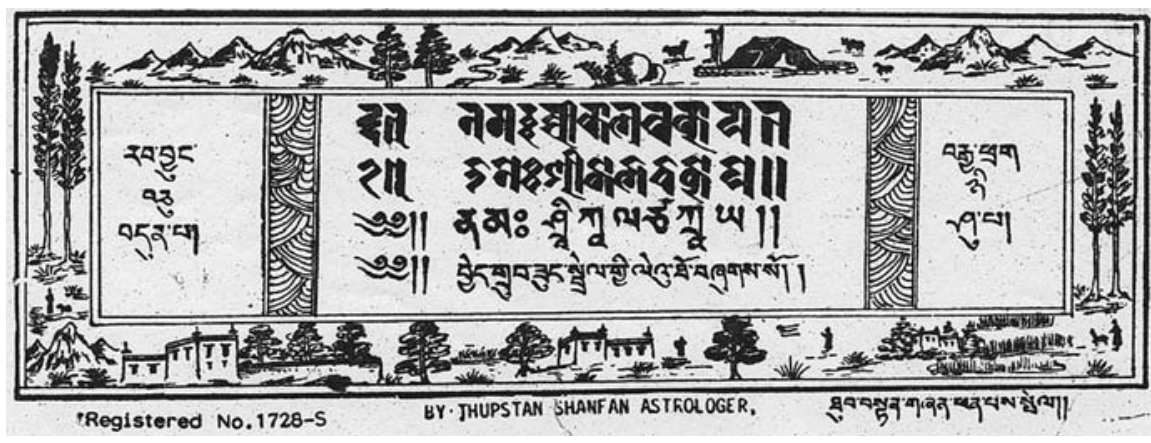

Figure 1. Couverture de l'almanach (le’u tho) de l'année 1996/97 correspondant aux années Souris de Feu et Bøuf de Feu du $17^{\mathrm{e}}$ cycle de soixante ans (rab byung).

10 Son titre complet est Byed grub zung sbrel kyi le'u tho. Les termes grub et byed renvoient à deux méthodes de calculs, relevant pour la première de l'école grub rtsis (ou phug lugs) fondée sur le 'Grel chen et pour la seconde de l'école byed rtsis fondée sur le Bsdus rgyud (Yamaguchi 1992).

11 Le Nouvel an (lo gsar), parfois appelé Nouvel an des agriculteurs (so nam pa'i lo gsar), est célébré au Ladakh un mois avant le Nouvel an tibétain, soit le premier jour du onzième mois tibétain. Sur le Nouvel an et le choix de cette date précoce, voir Dollfus (1987) et Khoo (1997).

12 Ses événements 'significatifs' concernent le développement et la transmission de la Doctrine bouddhique. 'Le plus important est le nirvana du Bouddha parce qu'il est le point de départ pour le calcul de la période pendant laquelle la Doctrine du Bouddha se perpétue.' (Imaeda 1984: 305) 
chacun; des diagrammes montrant la répartition des vingt-sept 'demeures lunaires' (rgyu skar, 'étoiles où se meuvent [les planètes]') ${ }^{13}$ sur le ventre et les membres de la Tortue d'Or, ${ }^{14}$ et leur association avec les neuf symboles: Joyau, Cheval, Ombrelle, Fouet, Lion, Eléphant, Livre sacré, Roue du Dharma, et Mudra de l'enseignement; des tableaux astrologiques, les uns de portée générale, les autres applicables aux individus.

La seconde partie donne des prévisions pour chaque mois. Y sont indiqués les éclipses de lune et de soleil, les jours 'coupés' ou 'redoublés', les jours fastes pour réaliser telle ou telle activité, et les jours 'brûlés' où toutes les actions entreprises sont vouées à l'échec (à l'exception des combats et des actes guerriers) Cornu (1999: 254).

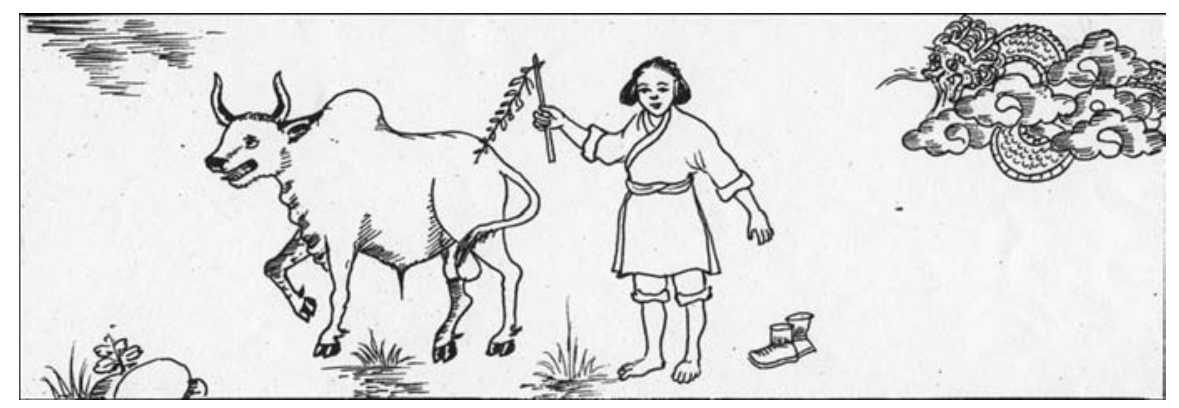

Figure 2. Dessin du bouvier (glang rdzi) illustrant les prévisions météorologiques de l'année.

${ }^{13}$ Ces constellations correspondent aux vingt-sept 'demeures lunaires' (skt. nakśátra) disposées en ceinture le long de la voûte céleste et traversées par la lune au cours de sa révolution autour de la terre. En tibétain, elles sont numérotées de 0 à 26 et nommées: 0. tha skar (skt. aśvin̄̄); 1. bra nye (skt. bharañ̄); 2. smin drug (skt. krttikā); 3 snar ma

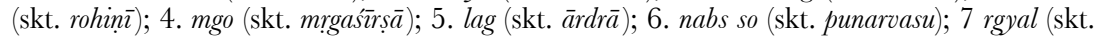
pusya); 8. skag (skt. āślesā). 9 mchu (skt. maghā); 10. gre (skt. pürva phālguñ̄); 11. dbo (skt. uttara phālguñ̄); 12. me bzhi (skt. hastā); 13. nag pa (skt. citrāa); 14 sa ri (skt. svātī); 15. sa ga (skt. višākhā); 16. lha mtshams (skt. anurādhāa); 17. snron (skt. jveșthā); 18. snrubs (skt. müla); 19. chu stod (skt. pūrva āsāạdhā); 20. chu smad (skt. uttara āsạdhāa); 21. gro bzhin (skt. Abhïit) et byi bzhin (skt. śravana); 22. mon gre (skt. śravișthā); 23. mon gru (skt. śătabhisāa); 24 'khrum stod (skt. pürva bhädrapada); 25 'khrum smad (skt. uttara bhädrapada); 26. nam gru (skt. revatī).

Ces noms ne sont pas les seuls usités. En effet, plusieurs de ces constellations sont connues sous deux ou trois appellations différentes. 'Les Pléiades' (smin drug), $3^{\mathrm{e}}$ constellation lunaire, est encore appelée mang po skyes et ma drug bu. Sur le nom des 27 ou 28 constellations en tibétain et en sanskrit, cf. Jacques (2007).

${ }^{14}$ La Tortue d'Or, gser gyi rus sbal, est la grande tortue cosmique qui forme la base de l'univers. On l'appelle aussi 'Celle qui a cinq membres', yan lag lnga pa. 


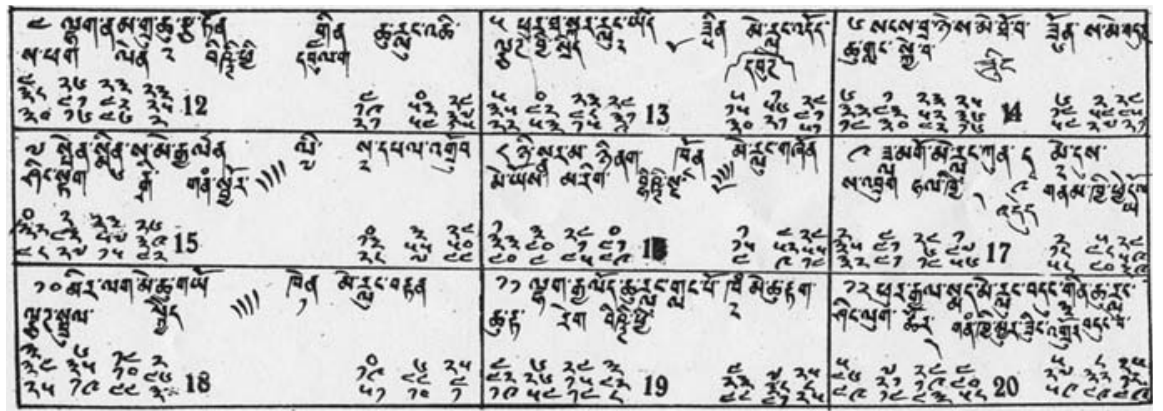

Figure 3. Page d'almanach couvrant la période du 12 au 20 mars 1997, 4 au $12^{\mathrm{e}}$ jour du second mois lunaire.

À chaque jour civil correspond une case rectangulaire offrant une profusion d'informations qui répondent à des usages variés et relèvent les unes de l'astrologie des éléments chinois ('byung rtsis), ${ }^{15}$ les autres de l'astrologie stellaire indienne (skar rtsis). ${ }^{16}$

On trouve ainsi:

- la date $\operatorname{ta}$ rig, ${ }^{17}$ donnée en chiffres arabes par le numéro du jour dans le calendrier grégorien, suivie pour le premier jour de chaque mois par le numéro du mois (1 pour janvier, 2 pour février, etc.);

- la date du jour du mois tibétain (tshes) donnée en chiffres tibétains;

- le jour de la semaine ( $\left.g z a^{\prime}\right)$ nommé d'après la planète qui le gouverne, l'élément (khams) lui correspondant et le numéro le désignant (0 pour samedi, 1 pour dimanche, 2 pour lundi, etc.);

- la 'demeure lunaire' (rgyu skar) et son élément;

- la grande conjonction résultant de la rencontre de la planète et de la demeure lunaire du jour;

- la combinaison élément-animal du jour;

${ }^{15}$ L'astrologie des éléments chinois s'apparente à la géomancie et se définit par l'observation des cycles d'énergie dans le temps.

${ }^{16}$ L'astrologie stellaire, fondée sur les mouvements des planètes dans l'espace céleste, prend en compte les positions relatives de la lune et des constellations.

${ }_{17} \mathrm{Du}$ hindi târikh: "date (in time); when preceded by a numeral, expresses that numbered day of the month in the European calendar" (Jäschke 1992: 280). 
- le trigramme spar $k h a^{18}$ et le dme $b a / m e w a^{19}$ du jour;

- le lien d'interdépendance rten 'brel:;

- des indications chiffrées renseignant sur la planète gouvernante et les positions relatives de la lune et du soleil, données à gauche pour la méthode de calculs de l'école grub pa, à droite pour la méthode de calculs de l'école byed $p a$;

- des informations sur la manifestation en ce jour de déités particulières, comme l'abréviation zin phung pour zin phung nag po, du nom d'une des principaux 'maîtres de la terre' dont l'influence astrologique est néfaste, ou les symboles signalant la sortie de - ou l'entrée en - retraite des divinités klu du bon vouloir desquelles dépendent la fertilité ou la stérilité des champs, l'abondance ou la pénurie. ${ }^{21}$

Dans l'exemple choisi (cf. Figure 4), la date est le 15 (mars 1997) dans le calendrier grégorien. Le jour du mois (tibétain) est le 7 (du second mois lunaire). Le jour de la semaine est le samedi-spen pour spen pa, Jour de Saturne, son chiffre est le 0, et son élément, la Terre $(s a) \cdot{ }^{22} \mathrm{La}$ demeure lunaire du jour est la constellation des Pléiades (smin drug) et l'élément lui correspondant le Feu, me.$^{23} \mathrm{La}$ grande conjonction est la bannière, rgyal mtshan. Le spar kha du jour est Li. Le dme ba du jour est le 7. La combinaison élément-animal du jour est Bois-Tigre (shing stag). Le lien d'interdépendance est 'la vieillesse et la mort' rgas shi, le dernier maillon de la coproduction conditionnée. Les nombres de 'l'étoile de la lune' (zla skar) et de 'l'étoile [visible pendant] le jour' (nyin skar) sont 2

18 Équivalent phonétique tibétain des huit pak' wa chinois à la base du $Y i$ king ou 'Livre des transformations', les spar kha (Li, Khon, Dva, Khen, Kham, Gin, Zin, Zon) jouent un grand rôle dans la géomancie. Contrairement aux mewa, ils sont liés à une direction fixe. Leur étude renseigne sur les directions favorables ou à éviter (Cornu 1999).

19 Relevant de la numérologie chinoise, les neuf mewa ou sme ba ('taches') forment les neuf cases d'un carré magique de trois. Chaque case est le palais d'une divinité du sol et se caractérise par une couleur, un chiffre, un élément et une direction. Sur les mewa/sme ba dans l'astrologie tibétaine, voir Hummel (1969) et Cornu (1999).

${ }^{20}$ Dans le bouddhisme, tous les phénomènes sont composés et interdépendants: "Ceci étant, cela devient; ceci apparaissant, cela naît [croît]. Ceci n’étant pas, cela ne devient pas; ceci cessant, cela cesse de naître [croître]." Cette coproduction conditionnée se présente comme un ensemble de douze liens, ou maillons, formant une suite cyclique: l'aveuglement; les créations mentales; la conscience discriminante; le nom et la forme, les six sphères sensorielles; le contact; la sensation; la soif; l'attachement; l'existence; la naissance; la vieillesse et la mort.

${ }_{21}$ Sur les divinités du sous-sol et du milieu aquatique, sa bdag et klu, et les croyances qui leur sont attachées au Ladakh, voir Dollfus (1996, 2002).

${ }^{22}$ thig spen pa sa'i khams

23 gnyis smin drug me'i khams. 

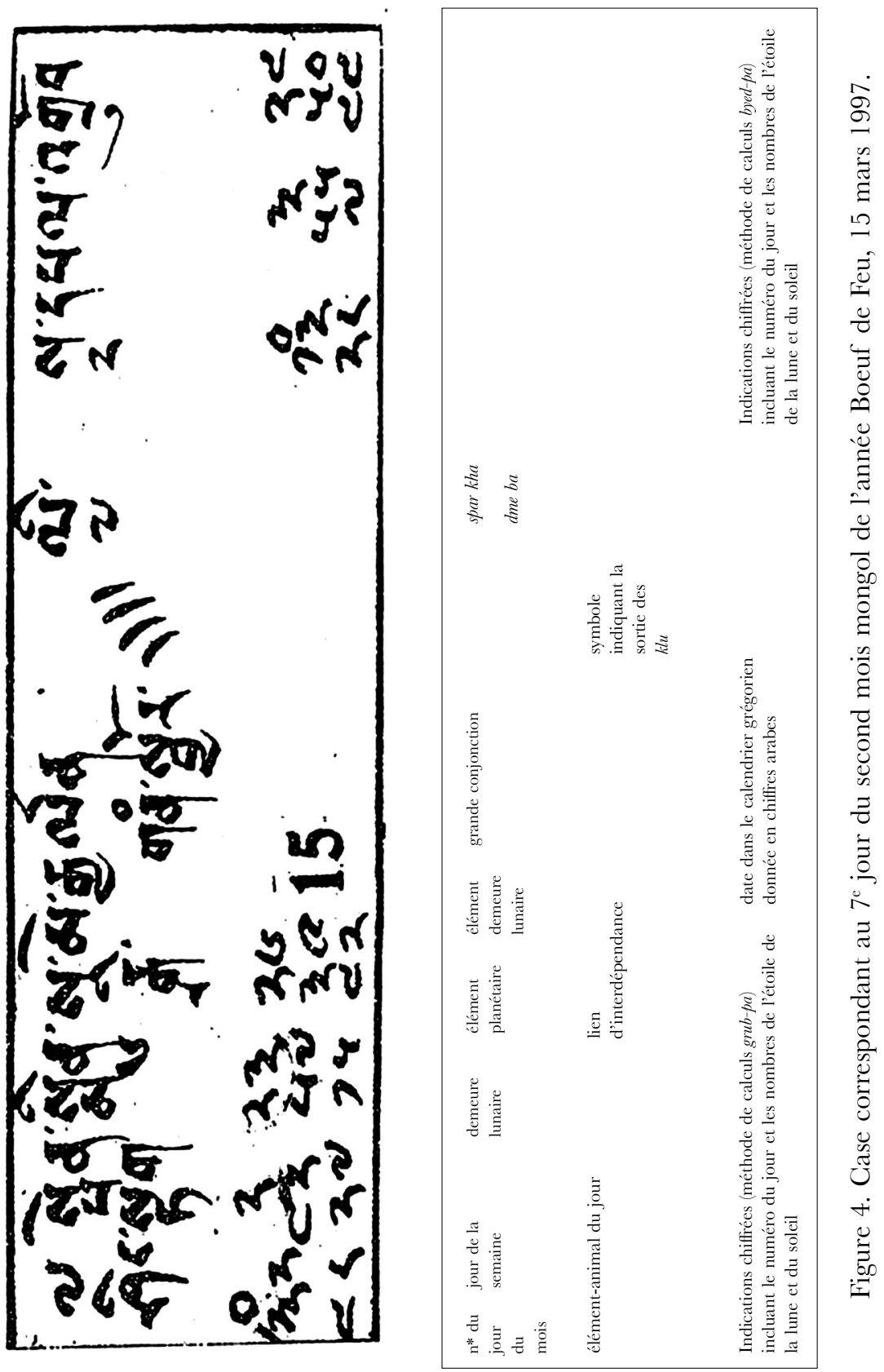
et 23 pour la méthode de calculs de l'école grub pa, 3 et 24 pour l'école byed $p a$. Enfin, quatre traits parallèles indiquent qu'il s'agit d'un jour de 'sortie des klu', klu thebs.

\section{Trouver le Four et L'heure Favorables}

Dans l'astrologie tibétaine, les jours possèdent des caractères généraux dont il faut tenir compte lorsqu'on recherche le meilleur jour pour mettre à exécution un projet ou prendre une décision importante. En effet, chaque jour de la semaine est gouverné par une planète, ellemême associée à un élément-Air, Eau, Feu ou Terre - déterminant par analogie les actions à entreprendre ou à éviter. Dans le cadre des travaux agricoles, ces relations de compatibilité ou d'incompatibilité sont rendues par la formule suivante: "Lundi, Mercredi, Vendredi et Samedi, sont bons; Dimanche et Mardi sont mauvais; Jeudi est neutre". ${ }^{24}$ Plus précisément, explique Onpo Gompo, le jeudi, présidé par Jupiter dont l'élément est l'Air (rlung), est un jour propice pour débuter le vannage; le lundi et le mercredi, associés à l'Eau (chu), sont favorables à l'irrigation, au contraire du dimanche et du mardi liés au Feu (me) et par conséquent 'mauvais' (ngan) pour tous les travaux en rapport avec l'eau, dont les semailles et les plantations; le vendredi et le samedi, associés à la Terre (sa), sont excellents pour les labours. Entre ces deux jours également fastes, l'astrologue opte pour le samedi, car ce jour de la semaine est réputé propice aux rituels de prospérité. Dans la quinzaine montante, il choisit le $7^{\mathrm{e}}$ jour du mois, plutôt que le $14^{\mathrm{e}}$, jour 'bon' (bzang) où tout acte violent doit être évité, dangereux pour travailler la terre, car y tuer un animal, même microscopique et fût-ce par inadvertance compte double. ${ }^{25}$

Un samedi, le $7^{\mathrm{e}}$ jour du mois, Onpo Gompo consulte l'almanach pour vérifier la pertinence de son choix. Il regarde d'abord si le jour pressenti ne correspond pas à un jour néfaste de 'retour des $k l u$ ', et note avec satisfaction qu'il est, au contraire, signalé par plusieurs traits parallèles comme un jour de 'venue des $k l u$ ', jour faste où ces divinités liées à la fécondité sont bien disposées à l'égard des hommes et prêtes à

24 zla ba lhag pa pa sang spen ba bzang/nyi ma dang mig mar ngan/phur bu dbye 'bring.

${ }^{25}$ Quand le cycle hebdomadaire (jours de la semaine positifs et négatifs) et le cycle mensuel s'opposent, seul le premier est pris en considération car les énergies planétaires sont considérées comme plus puissantes que les énergies mensuelles. 
les aider en libérant l'eau vive des torrents et des sources. C'est un bon présage pour une année de Feu placée sous le signe de la sécheresse. ${ }^{26}$ Il s'assure ensuite que la 'demeure lunaire', le mewa, le spar kha et la combinaison élément-animal du jour ne sont défavorables ni aux labours ni aux semailles. Puis il examine la grande conjonction résultant de la rencontre de la planète (Saturne) et de la constellation occupée par la lune ('les Pléiades'). Le résultat est 'la Bannière de Victoire' (rgyal mtshan): un pronostic excellent, garantissant biens et richesses en abondance.

D'ordinaire, une lecture attentive de l'almanach suffit à cerner la tonalité d'un jour et à juger de son adéquation avec l'acte à accomplir. Mais pour ce rituel d'importance par ses implications pour le Ladakh tout entier, de plus amples vérifications s’imposent.

Dans la case du jour, l'astrologue lit le nombre de 'l'étoile du soleil' (nyin skar), 'qui reste en place quatorze jours', et le nombre de 'l'étoile de la lune' (zla skar), 'qui évolue avec le disque solaire'; soit pour notre exemple: 23 et 2 dans le système grub pa, 24 et 3 dans le système byed pa. Puis, à l'aide des perles de son chapelet, il compte à voix haute combien les sépare: ' $23,24,25,26,27,28,{ }^{27} 1,2$ ', et reporte cette différence, ici 8 , sur la figure numérotée représentant un araire et un joug, placée en regard du texte intitulé 'Calculs pour ensemencer un champ' (zhing la sa bon 'debs pa'i rtsis).

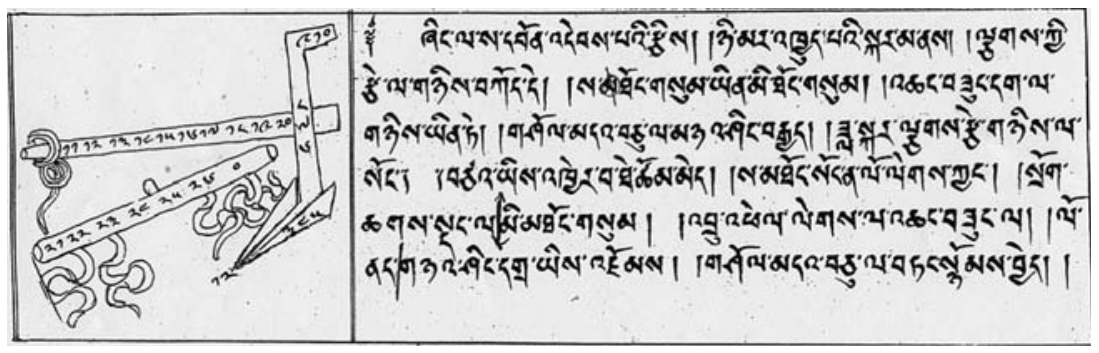

Figure 5. Figure numérotée représentant un araire et un joug. Dartsi, p. 151.

\footnotetext{
${ }^{26}$ Chaque année possède une tonalité générale en liaison avec l'élément et l'animal composant son signe. L'année de Terre est ainsi une année calme et propice à l'agriculture au contraire de l'année de Feu marquée par la sécheresse. L'année de la Souris est une année d'abondance où les greniers sont pleins comme les nids de ce petit rongeur; l'année du Bœuf est une année de labeur à l'image de cet animal obstiné, à la démarche lente et pesante.

${ }^{27}$ Le système comporte vingt-sept demeures lunaires, mais l'une d'elles se compose de deux constellations rapprochées (Cornu 1999: 169).
} 


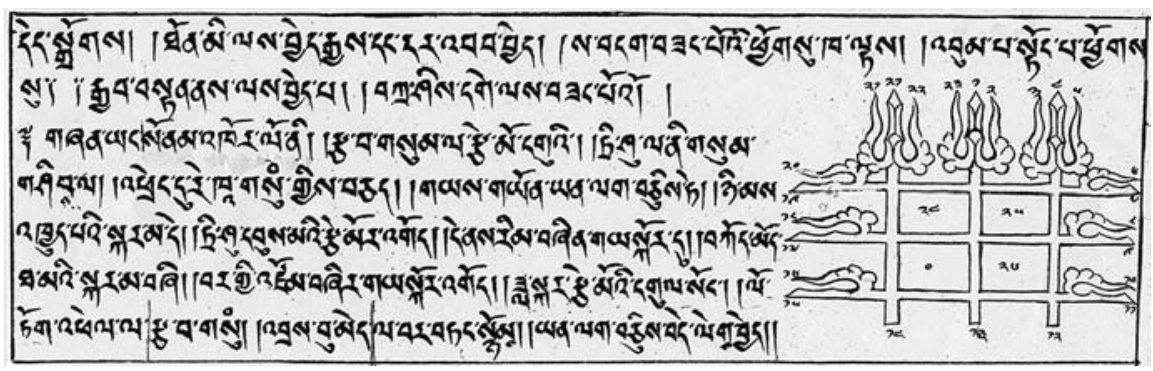

Figure 6. La roue des agriculteurs. Dartsi, p. 150.

Le chiffre 8 est situé dans la partie supérieure du sep, appelée mi thong, 'le sep de l'homme', par opposition à la partie inférieure enfouie dans le sol lors des labours et nommée sa thong, 'le sep de la terre'. Le commentaire dit: 'dans les trois du sep de l'homme [c'est-à-dire 6, 7 et 8], accroissement du grain' (mi thong gsum 'bru 'phel legs). Le présage est bon. Tel n'aurait pas été le cas, en revanche, si le calcul avait donné 9 (ou 10) et conduit dans la poignée de l'araire ('chang bzung), promettant 'des maladies' (lo nad), ou 21, 22, 23, 24, 25, ou 26 et conduit dans le joug (gnya' shing), annonçant 'la prise [des champs] par des ennemis' (dgra yis 'dzoms).

Onpo Gompo procède de même avec 'la roue des agriculteurs' (so nam 'khor lo): quatre rectangles juxtaposés, couronnés de trois 'tridents' (tri shu la) et bordés de douze 'branches' ( yan lag).

Ici, le nombre 8 désigne une des 'douze branches'. Le présage est heureux. En effet, il est écrit: 'dans les douze branches, le bien-être' ( yan lag bcu gnyis bde leg byed). Dans les pointes des trois tridents, l'augure était également favorable: 'multiplication de la récolte' (lo tog 'phel). En revanche, en leurs 'bases' (rtsa ba), numérotées 12, 13 et 14, le pronostic était mauvais: 'aucun fruit' ('bras bu med).

Notre astrologue recommence les mêmes calculs avec les nombres 24 et 3 relevant de l'école byed pa. Les résultats obtenus se révèlent concluants, confirmant que le septième jour du second mois mongol est une bonne date pour célébrer le rituel de 'la Bouche de la Terre'. Encore convient-il d'en préciser l'heure. En effet, pour établir les prédictions les plus justes, chaque division du temps - année, mois, jour et heure - doit être considérée à son échelon en respectant la hiérarchie évoquée par l'adage: 'l'an est roi, le mois ministre, le jour soldat et l'heure arme' (lo rgyal po zla ba blon po zhag dmag mi chu tshod mtshon cha). 


\begin{tabular}{|c|c|c|c|c|c|c|c|c|c|c|c|c|c|c|c|c|c|c|c|c|c|c|c|c|}
\hline \multirow{2}{*}{\multicolumn{3}{|c|}{ 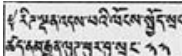 }} & & & & & के.5. & & भेष्बे & & 850 & ${ }^{3}$ & & 83 & & 점 & & $\Delta \bar{s}^{2}=$ & & 4.35 & & 4 & $d<2 N$ & \multirow{11}{*}{ 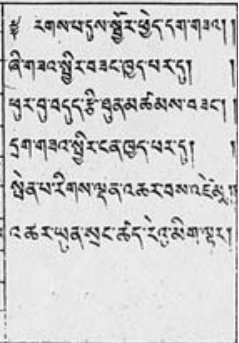 } \\
\hline & & & 눈 & की & 2 & ब्र & Ne & प्रे & ฉै & $\hat{\mathrm{B}} \mathrm{E}$ & $2 \widehat{\varepsilon}$ & है & 缕 & 8 & है। & \&ै & 这 & 里递 & & Q & र्? & है & \begin{tabular}{l|l} 
के & 5 \\
\end{tabular} & \\
\hline \multicolumn{3}{|c|}{ बशःतेरतु } & 5 & 6 & 7 & 8 & 9 & 10 & 111 & 12 & \begin{tabular}{l|l|}
1 & 2 \\
\end{tabular} & 3 & 4 & 5 & 6 & 7 & 8 & & & \begin{tabular}{l|l}
11 & 12
\end{tabular} & 1 & 2 & & \\
\hline ลิ & $58 \mathrm{~N}$ & 36 & 2 & 4 & 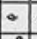 & 1 & शेल०० & बर्ज & 3 & 1. 5 & -4 & 3 & Eer & 1 & 5 ही & 1 & 4 & $42=$ & ज小० & $=0$ & $455^{2}$ & 3 & & \\
\hline 근 & $55^{\mathrm{N}}$ & 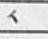 & 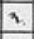 & aqu & 55? & 9 & , & 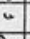 & $\div$ & 3 & $900-55$ & 3 & 1 & 3 & - & $\checkmark$ & 200 & $=1$ & 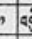 & 35 , & 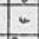 & , & Eित्र. & \\
\hline बैर & ब्रेजन & $\cdots$ & 4 & $n$ & 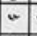 & - & 3 & रूने & $45^{2}$ & 71 & - & + & 3 & 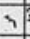 & and & 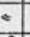 & ? & दूध & 4 & 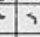 & $\hat{9}$ & - &, 905 & \\
\hline ชุๆ & बัศम & 3 & 4 & 3 & in & 45 & 4 & $?$ & 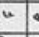 & 4 & $2 \times$ & 45 & 3 & 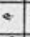 & ? & $855^{2}$ & 3 & 4 & & and- & ? & $8 \hat{5 \hat{f}^{\circ}}$ & 36 & \\
\hline 4ुz & $58 \mathrm{~N}$ & $8 \%$ & 95 & 4 & $n$ & 6 & + & 3 & रेनल & 155. & 1 & 6 & $=$ & 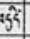 & $x$ & 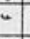 & 3 & हैल & $=$ & $195^{\circ}$ & 1 & 4 & $\rightarrow$ रे? & \\
\hline NEN & $4 \%$ & - & 4 & . & 3 & 2ans & 455 & 4 & 1 & 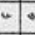 & 3 & $\operatorname{Ren} 9$ & 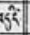 & $"$ & 3. & & * & 145 & & 4 & 3 & $E=\infty$ & 18 & \\
\hline छेन & 4:A & + & 250 & & 4 & & 4 & & 3 & & ह5 & 1 & 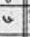 & & & & $45 \hat{~}$ & 34 & 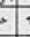 & $\hat{2}$ & $N=$ & , & $95 \%$ & \\
\hline \multicolumn{3}{|c|}{ 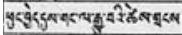 } & '9 & & 34 & & $x$ & & & & is & 16 & & & 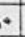 & & 4 & $1+1$ & & 装 & 1 & $y$ & & \\
\hline
\end{tabular}

Figure 7. Tableau récapitulatif des heures fastes et néfastes en fonction des jours de la semaine. Dartsi, p. 168.

Ouvrant une nouvelle fois le Dartsi, Onpo Gompo consulte le tableau des heures fastes et néfastes en fonction des jours de la semaine reproduit en page 168. Pour le Samedi (spen), les heures fastes, comparées à de l'ambroisie (bdud rtsi), sont: la première heure de l'aube (snga tho rengs), la seconde heure du lever du jour (phyi nam langs), la première heure de l'après-midi (snga phyed yol), la seconde heure du crépuscule (phyi sa sros). La troisième possibilité est retenue, car c'est la seule heure où il fasse jour à cette période de l'année.

\section{Déterminer le Lieu Propice et la Direction Pure}

La date et l'heure désormais fixées, il faut encore établir en quel point précis du champ faire porter 'la pointe en fer' du soc et dans quelle direction tracer le premier sillon. Pour cela, Onpo Gompo s'intéresse aux 'maîtres de la terre' (sa bdag) ${ }^{28}$ et, plus spécialement, à l'un d'eux décrit dans les textes astrologiques sous le nom de 'maître de la terre qui rampe sur le ventre' (sa bdag lto 'phye), ${ }^{29}$ et connu familièrement des Ladakhi sous l'expression homophone de 'maître de la terre au gros ventre' (sa bdag lto che). Le Dartsi ${ }^{30}$ le dépeint de couleur jaune, avec la tête d'un taureau surmontée de deux cornes (d'or à droite, de turquoise à gauche), le corps d'un homme et la queue d'un serpent. Il tire une langue de feu et porte un chaperon turquoise formé de sept serpents.

${ }^{28}$ Sur les sa bdag, divinités d'une bienveillante neutralité, mais qui peuvent envoyer malheurs et maladies si elles se sentent agressées, voir Dollfus (1996, 2003).

${ }^{29}$ Ce serpent joue un rôle fondamental dans 'le rituel du sol', sa chog, préalable indispensable au creusement des fondations d'un temple, d'un stupa, d'un palais (Gardner 2005-6).

30 'Bras rtsis: 260-61. 


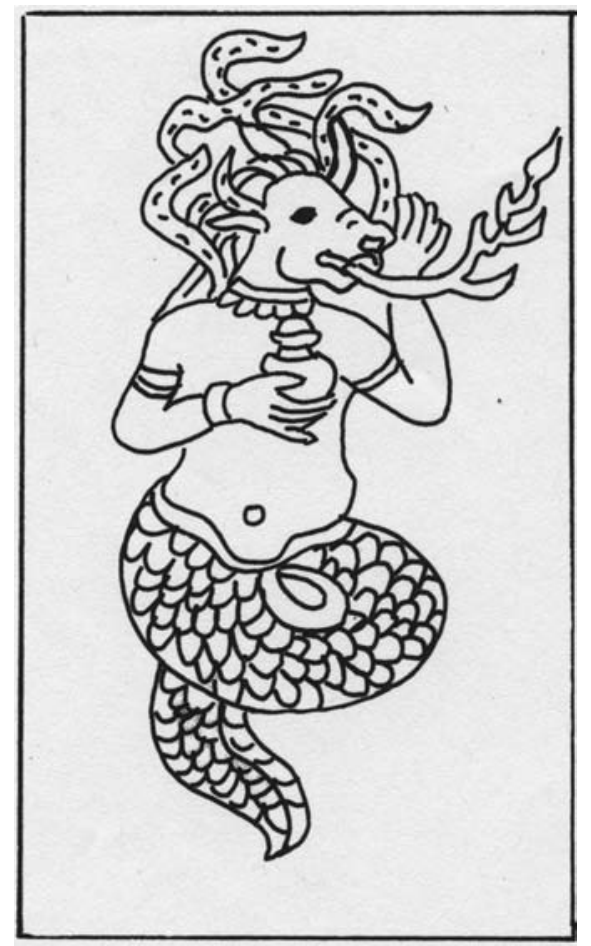

Figure 8. Le 'maître de la terre qui rampe sur ventre'. (Dessin tiré du Dartsi)

Penché en avant, il couvre son nez de sa main gauche et tient un vase rempli de précieux joyaux dans sa main droite.

Il ne reste pas immobile, mais se déplace sous la terre au rythme des saisons. Pendant les trois mois d'été, sa tête est à l'ouest, sa queue à l'est, son ventre au nord et son dos au sud; pendant les trois mois d'automne, sa tête est au sud, sa queue au nord, son ventre à l'ouest et son dos au nord; pendant les trois mois d'hiver, sa tête est à l'est, sa queue à l'ouest, son ventre au sud et son dos au nord; pendant les trois mois de printemps, sa tête est au nord, sa queue est au sud, son ventre à l'est et son dos à l'ouest. Il est prédit la mort d'un père, d'une mère ou d'un fils à celui qui heurte sa tête; disputes et conflits à celui qui heurte ses cornes d'or et de turquoise; la fuite des richesses à celui qui heurte sa queue. En revanche, l'abondance est promise à celui qui heurte le ventre de ce serpent et brise le vase qu'il tient par devers lui, répandant ainsi son contenu dans la terre. C'est donc en cet endroit précis, situé à l'est au '[mois] médian du printemps' (dpyid 'bring ba), que le soc de l'araire doit ouvrir la terre. 


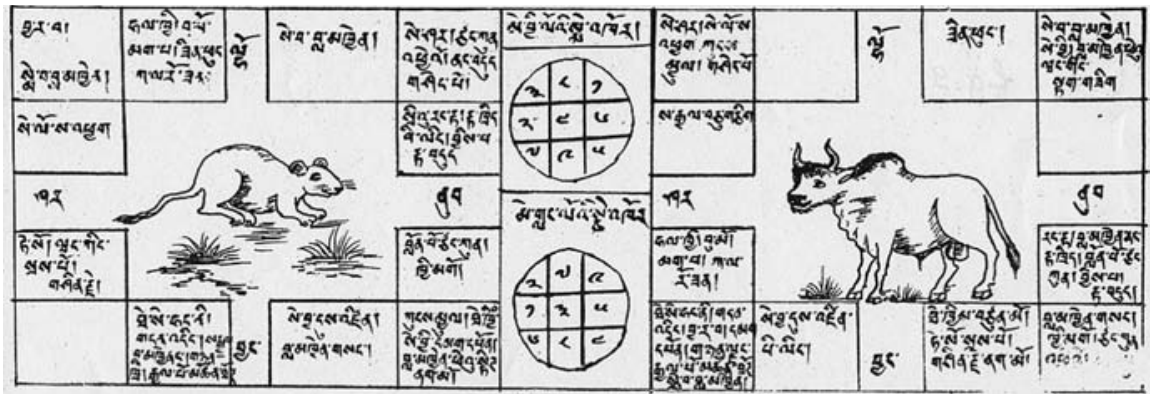

Figure 9. Diagramme de répartition des maîtres de la terre pour l'année de la Souris (à gauche) et l'année du Bœuf (à droite).

Pour déterminer la direction dans laquelle creuser la première raie (rol ka), l'astrologue considère la position d'autres 'maîtres de la terre', notamment celles de The-se, le roi des sa bdag du cycle duodénaire, et des membres de sa cour. Dans l'almanach, un diagramme, accompagné de commentaires sur la conduite à tenir, indique les directions qu'ils occupent en fonction du signe de l'année (lo khams).

L'année du Bœuf, The-se réside au Nord-Est, orient de l'animal gouverneur; son épouse The-khyim et son fils Te-so au Nord Supérieur; son garde du corps Se-shar au Sud-Est; son astrologue Se-ba bla-ma mkhyen et son gardien des trésors Se-byi au Sud-Ouest; son ministre Tsang-kun, monté sur une tortue, au Nord-Ouest; son coursier Rangrta et son écuyer Rta-khrid à l'Ouest inférieur; son serviteur Hal-khyi à l'Est supérieur. Quant à Zin-phung nag-po, le maître de la terre aux pinces de scorpion, il occupe le Sud Inférieur. En conséquence, 'les directions pures, sans sa bdag, où petits et grands travaux peuvent être entrepris, sont le Sud supérieur (lho stod) et l'Ouest supérieur (nub stod), ${ }^{31}$ deux directions marquées sur le diagramme par des rectangles vides de toute inscription. Pour choisir la plus favorable, Onpo Gompo procède à l'examen du 'vase vide' (bum stong), dont l'orientation est liée au signe du zodiaque (khyim). Or, il est écrit dans l'almanach de l'année: 'du 19 e jour du second mois au $20^{\mathrm{e}}$ jour du troisième mois, le vase vide [ouvre à] l'ouest'. Pour éviter de le remplir avec les richesses de la terre, cette direction doit donc être écartée.

31 sa bdag med pa'i phyogs gtsang ni lho stod nub stod gnyis pa che phra'i las 'gro ba brtsam par bya. 


\section{Choisir les Bêtes de Labour, L'homme Guidant L'attelage, le Laboureur et le Semeur}

Pour sélectionner bêtes et hommes, l'astrologue ne s'intéresse plus à la tonalité du jour, mais aux caractéristiques propres à l'année en cours. Sont prises spécialement en compte les cinq forces spécifiques à l'astrologie tibétaine et de première importance dans l'établissement des horoscopes: la Vitalité (srog), le Corps (lus) ou énergie de la santé physique, le Pouvoir (dbang thang) ou habileté à réaliser ses buts, ${ }^{32}$ l'Âme (bla) ou état psychique, et le 'Cheval du Souffle' (rlung rta) ${ }^{33}$ ou Chance. Chacune de ces forces est associée à un element-Bois, Feu, Terre, Eau ou Fer - qui entretient avec les autres des relations de type mère $(m a)$, fils $(b u)$, ami (grogs) et ennemi (dgra), obéissant à des règles précises développées dans 'Les Rayons de la Lune, Préceptes de l'Astrologie des Éléments'. ${ }^{34}$

Le Bois - explique ainsi Onpo Gompo - est la mère du Feu qu'il engendre, l'Eau est la Mère du Bois qu'elle nourrit, la Terre est la mère du Fer que l'on extrait de ses profondeurs... En revanche, le Feu est l'ennemi du Bois qu'il brûle, le Fer est l'ennemi du Bois qu'il coupe, l'Eau est l'ennemi du Feu qu'elle éteint.

Dans le Dartsi, des tableaux donnent les éléments correspondant aux cinq forces pour les derniers cycles de soixante ans (rab byung). ${ }^{35}$ Pour l'année Bœuf de Feu (1997), l'élément de la Vitalité est la Terre, l'élément du Corps est l'Eau, l'élément du Pouvoir est le Feu, l'élément de l'Âme est le Feu, l'élément du Cheval du Souffle est l'Eau.

Pour le choix des bêtes de labour, c'est ce dernier élément qui est retenu car - indique notre astrologue - le klung rta est la mère de toutes les autres énergies et leur principe d'harmonisation. Cet element-l'Eau pour l'année Bœuf de Feu-conditionne la couleur de leur robe. L'Eau

\footnotetext{
${ }^{32}$ Les Ladakhi lui donnent le sens de 'situation économique'.

33 Orthographié klung rta dans les textes astrologiques. Sur cette notion, voir Cornu (1999).

34 'byung rtsis man ngag zla ba'i 'od gzer, ouvrage traitant de l'astrologie des éléments selon la tradition chinoise écrit par Lochen Dharmashri (1654-1717), et voir Cornu (1999: 67-77). Dans la pensée chinoise, contrairement à ce qu'affirme la théorie indienne, les éléments s'organisent en un système dynamique de cycles de création et de destruction.

${ }^{35}$ L'ère rab byung commence en 1026 de notre ère, au moment de l'adoption du système indien. Le $17^{\mathrm{e}}$ cycle correspond aux années 1987-2046.
} 


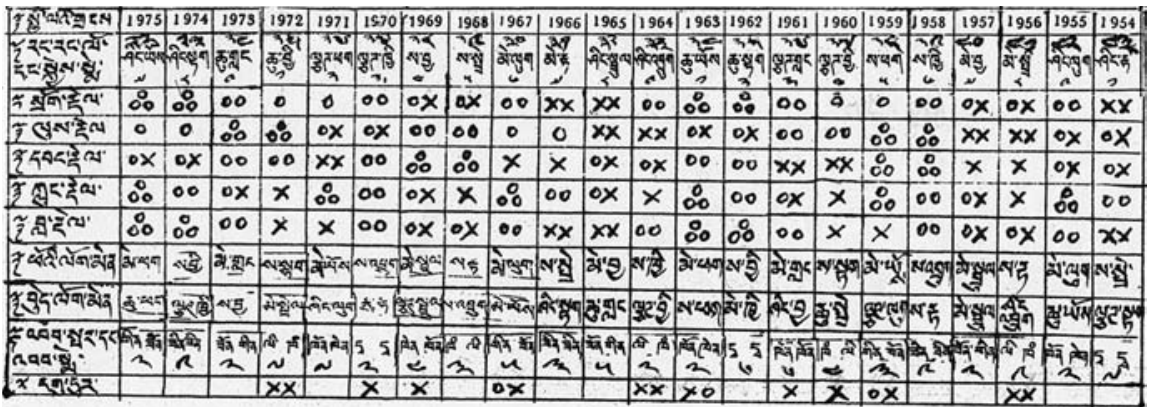

Figure 10. Tableau comparant les éléments des cinq énergies de l'année aux éléments de naissance.

étant associée au bleu (sngon po), les bovins qui tractent l'araire doivent être bleus.

Pour les hommes, l'astrologue examine leur condition et leur âge ou, plus exactement, leur année de naissance. Pour appeler la chance et la prospérité sur l'année agricole à venir, ils doivent être en bonne santé, 'posséder père et mère' (pha ma tshang mkhan), mais être suffisamment âgés pour 'savoir' (shes mkhan). En outre, les trois principaux protagonistes - le conducteur de l'attelage, le laboureur et le semeur - doivent remplir les conditions énoncées dans les 'Calculs pour ouvrir la Bouche de la Terre' (sa kha phyed pa'i rtsis) et les 'Calculs pour dompter les nouveaux [ou les grands] bœufs' (glang gsar [glang chen] 'dul ba'i rtsis). L'élément de Vitalité de l'année doit ainsi être la mère de l'élément de naissance de l'homme qui tire les $m d z o$ (hybrides de yak et de vache); l'élément du Pouvoir de l'année, l'ennemi de l'élément de naissance de l'homme qui tient l'araire mais la mère de l'élément de naissance du semeur.

Pour trouver ces individus, Onpo Gompo étudie les tableaux comparant les éléments des cinq énergies de l'année aux éléments de naissance, imprimés en première partie de l'almanach. Les degrés d'affinité ou d'inimitié y sont figurés respectivement par des cercles (o), dits 'petits cailloux blancs' (rdel dkar), ou des croix noires (x), appelées 'petits cailloux noirs' (rdel nag).

La relation maternelle, excellente, est rendue par trois cercles; la relation amicale, bonne, par deux cercles; la relation filiale, neutre, par un cercle et une croix; et la relation d'inimitié, néfaste, par deux croix.

Pour l'année Bœuf de Feu, le résultat est le suivant: le conducteur de l'attelage doit être né une année Tigre de Bois, le laboureur une 
année Oiseau de Fer, le semeur une année Cheval de Terre, soit en 1974, 1981 et 1978.

La nuit tombe et le travail de l'astrologue touche à son terme. Il ne lui reste plus qu'à rédiger un aide-mémoire et à le remettre au chef du quartier de Leh, responsable cette année de l'organisation de la cérémonie du premier labour. Sur une page de cahier d'écolier, Onpo Gompo écrit en caractères d'imprimerie:

Om sva sti.

Salut à vous!

Voici les calculs pour l'ouverture de la Bouche de la Terre par les agriculteurs.

Année de Feu; année du Bœuf.

Septième jour du second mois lunaire.

Samedi à une heure, la moitié de la journée écoulée.

À une heure sur la montre.

[Tracer] la première raie dans la direction pure du Sud Supérieur.

Des $m d z o$ de couleur bleue.

Dans le 'sep de l'homme', du bon grain en abondance selon l'araire et la roue [des agriculteurs].

Pour conduire les $m d z o$ par le nez, un homme de l'année Tigre de Bois, 24 [ans].

Pour tenir l'araire, un homme de l'année Oiseau de Fer, 17 [ans].

Pour verser les semences, un homme de l'année Cheval de Terre, 20 [ans].

Libations (gser skyems, 'breuvage d'or') et [lecture des textes] gnam sa snang rgyas et bkra shis rtsegs $p a^{36}$ sont recommandés.

Sonam Gompo est mort en 2003 d'une cirrhose alcoolique. Évoquer sa mémoire incite à réfléchir sur la transmission des savoirs, le devenir de l'astrologie et, plus largement, sur le futur de ce type de rituels dans une région qui, à l'ère de la mondialisation, connaît de profonds changements. Comme il le pressentait, aucun des siens n'a pris la relève. Son unique fils a choisi une autre voie: suivant l'exemple de nombreux jeunes de sa génération, il a intégré le corps de l'armée indienne. Thupstan Shanfan est aujourd'hui le seul spécialiste en astrologie des éléments (nag rtsis) de la région de Leh. Il continue de publier calendriers et

\footnotetext{
${ }^{36}$ Ces textes de bénédiction d'usage courant, explique Onpo Gompo, servent d'antidotes en cas d'erreur dans l'exécution du rituel. Le premier s'adresse aux maîtres de la terre dont la tranquillité va être perturbée, le second est lu pour accroître le bonheur, le bien-être et la chance; tous deux sont accompagnés de libations d'alcool.
} 
almanachs, mais rares sont aujourd'hui ceux capables d'en interpréter les informations. Le rituel du premier sillon avec ses yaks au front blanchi et aux cornes huilées fait désormais partie du folklore ladakhi avec le tir à l'arc, le polo, ou les mascarades du Nouvel an, autant de spectacles (ltad mo) qualifiés de 'bigarrés et pittoresques'. Des bribes en sont données à voir - hors contexte - à l'occasion du festival du Ladakh qui se tient à Leh au cours de la première quinzaine de septembre, et attire davantage de spectateurs chaque année.

\section{Bibliographie}

\section{Textes Tibétains}

Thub bstan gshan phan, 1985. 'Bras rtsis gsar phreng kun dga'i snying nor. (Leh, Ladakh). Thub bstan gshan phan, 1976. Byed grub zung sbrel gyi le'u tho (Leh, Ladakh).

\section{Littérature Secondaire}

Berzin, Alexander. 1987. An introduction to Tibetan astronomy and astrology. The Tibet Fournal 12(1): 17-28. The Berzin Archives. http://www.berzinarchives.com/tibetan Cornu, Philippe. 1999. L'astrologie tibétaine. Paris: Guy Trédaniel.

Dollfus, Pascale. 1987. Lo-gsar, le Nouvel An populaire au Ladakh. L'Ethnographie 83: 63-96.

- 1990. De l'ordre et de la prospérité: analyse de deux rituels agraires au Ladakh. Dans Wissenschaftsgeschichte und gegenwärtige Forschungen in Nordwest-Indien, sous la dir. de G. Meier et P. Neumann, Dresden, Staatliches Museum für Völkerkunde Dresde Forschungstelle: $220-32$.

- 1996. Maîtres du sol et dieux du territoire au Ladakh. Etudes rurales 143-44: $27-44$.

2003. De quelques histoires de klu et de btsan. Revue d'Etudes Tibétaines (RET) 2: 4-39. http://www.digitalhimalaya.com/ret/RET_2.pdf.

Gardner, Alexander. 2005-6. The sa chog. Violence and veneration in a Tibetan soil ritual. Etudes Mongoles et Sibériennes, Centrasiatiques et Tibétaines 36-37: 283-324.

Hummel, Siegbert. 1969. The sme ba dgu, the magic square of the Tibetans. East and West 19(1-2): 139-46.

Imaeda, Yoshiro. 1984. Mémento chronologique (Bstan rtsis) du Calendrier bhoutanais. Dans L. Ligeti (éd.), Tibetan and Buddhist Studies commemorating the 200th anniversary of the Birth of A. Csoma. de Köros. Budapest, vol. 1, 303-20.

Jacques, Guillaume. 2007. Le nom des naksatrani en tibétain. Revue d'Etudes Tibétaines (RET ) 12: 4-10. http://www.digitalhimalaya.com/collections/journals/ret/index. php

Jäschke, H.A. 1980 [1881]. A Tibetan-English Dictionary with Special Reference to the Prevailing Dialects. Delhi/Varanasi/Patna: Motilal Banarsidass.

Khoo, Michael. 1997. Preliminary Remarks concerning Solar Observation, Solar Calendars and Festivals in Ladakh and the Western Himalaya. Dans T. Dodin et R. Heinz (éds), Recent Research on Ladakh 7. Ulm: Ulmer Kulturanthropologische Schriften, 235-70.

Lafitte, Jean-Jacques. 1985. Le calendrier tibétain. La Nouvelle Revue Tibétaine 12: 24-31. 
Riaboff, Isabelle. 1997. Notes sur les rituels agraires au Zanskar: terre, terroirs, territoires. Dans H. Krasser, M. Torsten Much, E. Steinkellner et H. Tauscher (éds.), Tibetan studies: Proceedings of the 7th Seminar of the International Association for Tibetan Studies, Graz Vol. II, Vienne, Verlag der Österreichischen Akademie der Wissenschaften: 803-16. - 2000. Jeux printaniers au Zanskar: Le tir à l'arc de Zangla: Bouffons et héros, rois et étrangers. Etudes mongoles et sibériennes 30-31: 137-79.

Yamaguchi, Zuiho. 1992 The significance of intercalary constants in the Tibetan calendar and historical tables of intercalary months. Dans Shohen Ihara et Zuiho Yamaguchi (éds), Tibetan Studies, Proceedings of the 5th Seminar of the International Association of Tibetan Studies, Narita 1989. Narita: Naritasan Shishoji, 873-95. 